International Journal of Pure and Applied Mathematics

Volume 100 No. 1 2015, 53-57

ISSN: 1311-8080 (printed version); ISSN: 1314-3395 (on-line version)

url: http://www.ijpam.eu

doi: http://dx.doi.org/10.12732/ijpam.v100i1.5

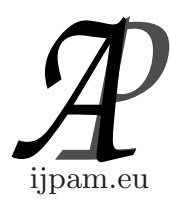

\title{
STABILITY OF LIE HOMOMORPHISMS ON BANACH ALGEBRAS
}

\author{
B. Yousefi ${ }^{1 \S}$, Gh. Moghimi ${ }^{2}$ \\ ${ }^{1,2}$ Department of Mathematics \\ Payame Noor University \\ P.O. Box 19395-3697, Tehran, IRAN
}

\begin{abstract}
In this paper we prove the Hyers-Ulam stability of special homomorphisms on some Banach algebras associated to a generalized functional equation.
\end{abstract}

AMS Subject Classification: 39B52, 39B82, 46B99

Key Words: $n$-ary homomorphism, Hyers-Ulam Stability, n-ary Banachalgebra, Functional equation, Lie product

\section{Introduction}

A classical question in the theory of functional equations is: When is it true that a function which approximately satisfies a functional equation must be close to an exact solution of the equation? If the problem accepts a solution, we say that the equation is stable. For some sources see [1-4].

Definition 1.1. An n-ary algebra $A$ is a complex linear space, endowed with an n-array product $\left(x_{1}, \ldots, x_{n}\right) \rightarrow\left[x_{1} \ldots x_{n}\right]_{A}$ from $A^{n}$ into $A$ such that

$$
\begin{aligned}
{\left.\left[x_{1} \ldots x_{n}\right]_{A} y_{1} \ldots y_{n-1}\right]_{A} } & =\left[x_{1}\left[x_{2} \ldots x_{n} y_{1}\right]_{A} y_{2} \ldots y_{n-1}\right]_{A}=\ldots \\
& =\left[x_{1} \ldots x_{n-1}\left[x_{n} y_{1} \ldots y_{n-1}\right]_{A}\right]_{A}
\end{aligned}
$$

for all $x_{1}, \ldots, x_{n}, y_{1}, \ldots, y_{n-1}$ in $A$.

Received: November 18, 2014

(C) 2015 Academic Publications, Ltd.

${ }^{\S}$ Correspondence author 
Definition 1.2. A Banach algebra $A$, endowed with the Lie product $[x, y]:=(x y-y x) / 2$ on $A$, is called a Lie Banach algebra.

Definition 1.3. Assume that the algbras $A$ and $B$ are complex n-ary algebras. A linear mapping $H: A \rightarrow B$ is said to be an n-ary Lie homomorphism if

$$
\begin{aligned}
& H\left(\left[x_{1} \ldots x_{n}\right]_{A}\right)=\left[H\left(x_{1}\right) \ldots H\left(x_{n}\right)\right]_{B},
\end{aligned}
$$

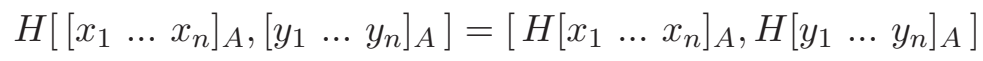

hold for all $x_{1}, \ldots, x_{n}$ in $A$.

We want to investigate the Hyers-Ulam stability of n-ary Lie homomorphisms.

\section{Hyers-Ulam Stability of $\boldsymbol{n}$-ary Homomorphisms}

In this section, we want to investigate the Hyers-Ulam stability of n-ary Lie homomorphisms acting on n-ary Lie Banach algebras associated with the following generalized functional equation:

$$
\begin{array}{r}
\mathrm{f}\left(\frac{\sum_{i=1}^{n} x_{i}}{n+1}\right)+\mathrm{f}\left(\frac{n x_{1}-\sum_{i=2}^{n-1} x_{i}-(n+1) x_{n}}{n+1}\right)+\mathrm{f}\left(\frac{(n+1) x_{1}+n x_{n}}{n+1}\right) \\
-2 \mathrm{f}\left(x_{1}\right)=0
\end{array}
$$

For a given mapping $g: A \rightarrow B$, we define

$$
\begin{aligned}
& C_{\mu} \quad g \quad\left(x_{1}, \ldots, x_{n}, y_{1}, \ldots, y_{n}, z_{1}, \ldots, z_{n}, w_{1}, \ldots, w_{n}\right) \\
& =\frac{1}{\mu} g\left(\frac{\mu x_{2}+\sum_{i=1, i \neq 2}^{n} x_{i}}{n+1}\right)+g\left(\frac{n x_{1}-\sum_{i=2}^{n-1} x_{i}-(n+1) x_{n}}{n+1}\right) \\
& +g\left(\frac{(n+1) x_{1}+n x_{n}}{n+1}\right)-2 g\left(x_{1}\right)+g\left(\left[y_{1} \ldots y_{n}\right]_{A}\right)-\left[g\left(y_{1}\right) \ldots g\left(y_{n}\right)\right]_{B} \\
& +g\left[\left[\begin{array}{lll}
z_{1} & \ldots & z_{n}
\end{array}\right]_{A},\left[\begin{array}{lll}
w_{1} & \ldots & w_{n}
\end{array}\right]_{a}\right]-\left[g\left[\begin{array}{lll}
z_{1} & \ldots & z_{n}
\end{array}\right]_{A}, g\left[\begin{array}{lll}
w_{1} & \ldots & w_{n}
\end{array}\right]_{A}\right] \text {. }
\end{aligned}
$$

Theorem 2.1. Assume that $\left(A,[]_{A}\right)$ and $\left(B,[]_{B}\right)$ are n-ary Lie Banach algebras and $n \geq 3$. Suppose that $f: A \longrightarrow B$ be an odd function satisfying: 


$$
\begin{array}{r}
\left\|C_{\mu} f\left(x_{1}, \ldots, x_{n}, y_{1}, \ldots, y_{n}, z_{1}, \ldots, z_{n}, w_{1}, \ldots, w_{n}\right)\right\| \\
\quad \leq \varphi\left(x_{1}, \ldots, x_{n}, y_{1}, \ldots, y_{n}, z_{1}, \ldots, z_{n}, w_{1}, \ldots, w_{n}\right),
\end{array}
$$

for all $\mu \in \mathbb{C}$ with $|\mu|=1$ and all $x_{1}, \ldots, x_{n}, y_{1}, \ldots, y_{n}, z_{1}, \ldots, z_{n}, w_{1}, \ldots, w_{n}$ in $A$, where $\varphi: A^{4 n} \longrightarrow[0, \infty)$ is a function such that

$$
\begin{aligned}
\varphi\left(2 x_{1}, \ldots, 2 x_{n}, 2 y_{1}, \ldots, 2 y_{n}, 2 z_{1}, \ldots, 2 z_{n}, 2 w_{1}, \ldots, 2 w_{n}\right) \\
\leq 2 r \varphi\left(x_{1}, \ldots, x_{n}, y_{1}, \ldots, y_{n}, z_{1}, \ldots, z_{n}, w_{1}, \ldots, w_{n}\right),
\end{aligned}
$$

for some $0<r<1$ and all $x_{1}, \ldots, x_{n}, y_{1}, \ldots, y_{n}, z_{1}, \ldots, z_{n}, w_{1}, \ldots, w_{n} \in A$. If

$$
\lim _{m, k \rightarrow \infty} \frac{1}{2^{m}} \sum_{i=1}^{k} \frac{1}{2^{i}} \varphi\left(0, \ldots, 0,2^{i+m-1} x_{2}, \ldots, 2^{i+m-1} x_{n}, 0, \ldots, 0\right)=0,
$$

for all $x_{2}, \ldots, x_{n} \in A$, then there exists a unique an n-ary Lie homomorphism $H: A \longrightarrow B$ such that

$$
\begin{aligned}
& \|f(x)-H(x)\| \\
& \leq \sum_{i=1}^{\infty} \frac{1}{2^{i}} \varphi\left(0,2^{i-1}\left(\frac{n+1}{n}\right) x_{2}, \ldots, 2^{i-1}\left(\frac{n+1}{n}\right) x_{n-1}, 2^{i-1}\left(\frac{n+1}{n}\right) x, 0, \ldots, 0\right),
\end{aligned}
$$

for all $x, x_{2}, \ldots, x_{n-1} \in A$ satisfying $(1+2 n) x=-\left(x_{2}+\ldots+x_{n-1}\right)$.

Proof. In (2), put $\mu=1, x_{1}=y_{1}=\ldots=y_{n}=z_{1}=\ldots=z_{n}=w_{1}=\ldots=$ $w_{n}=0$. Then we get

$$
\left\|C_{1} \mathrm{f}\left(0, x_{2}, \ldots, x_{n-1}, x_{n}, 0, \ldots, 0\right)\right\| \leq \varphi\left(0, x_{2}, \ldots, x_{n-1}, x_{n}, 0, \ldots, 0\right)
$$

for all $x_{2}, \ldots, x_{n-1}, x_{n} \in A$ satisfying $(1+2 n) x_{n}=-\left(x_{2}+\ldots+x_{n-1}\right)$. Hence

$$
\left\|\mathrm{f}\left(\frac{n}{n+1} x_{n}\right)-\frac{1}{2} \mathrm{f}\left(2\left(\frac{n}{n+1} x_{n}\right)\right)\right\| \leq \frac{1}{2} \varphi\left(0, x_{2}, \ldots, x_{n}, 0, \ldots, 0\right)
$$

for all $x_{2}, \ldots, x_{n-1}, x_{n} \in A$ satisfying $(1+2 n) x_{n}=-\left(x_{2}+\ldots+x_{n-1}\right)$. By proceeding in this way, we obtain

$$
\begin{aligned}
\| f\left(\frac{n}{n+1} x_{n}\right)-\frac{1}{2^{k}} \mathrm{f}\left(2^{k}\left(\frac{n}{n+1} x_{n}\right)\right) & \\
& \leq \sum_{i=1}^{k} \frac{1}{2^{i}} \varphi\left(0,2^{i-1} x_{2}, \ldots, 2^{i-1} x_{n}, 0, \ldots, 0\right),
\end{aligned}
$$


for all $x_{2}, \ldots, x_{n-1}, x_{n} \in A$ satisfying $(1+2 n) x_{n}=-\left(x_{2}+\ldots+x_{n-1}\right)$. Replace $x_{n}$ by $2^{m} x_{n}$ in (6) and then divide by $2^{m}$, we get

$$
\begin{aligned}
\| \frac{1}{2^{m}} \mathrm{f}\left(2^{m}\left(\frac{n}{n+1} x_{n}\right)\right) & -\frac{1}{2^{m+k}} \mathrm{f}\left(2^{m+k}\left(\frac{n}{n+1} x_{n}\right)\right) \| \\
& \leq \frac{1}{2^{m}} \sum_{i=1}^{k} \frac{1}{2^{i}} \varphi\left(0,2^{i+m-1} x_{2}, \ldots, 2^{i+m-1} x_{n}, 0, \ldots, 0\right),
\end{aligned}
$$

for all integers $m, k$ and all $x_{2}, \ldots, x_{n-1}, x_{n} \in A$ satisfying $(1+2 n) x_{n}=-\left(x_{2}+\right.$ $\left.\ldots+x_{n-1}\right)$. By the relations (4) and (7), the sequence $\left\{\frac{1}{2^{m}} \mathrm{f}\left(2^{m}\left(\frac{n}{n+1}\right) x_{n}\right)\right\}_{m}$ is a Cauchy sequence in $\mathrm{B}$, for all $x_{n} \in A$, so it is convergent. Thus there exists $H: A \rightarrow B$ such that $H(x)=\lim _{m \rightarrow \infty} \frac{1}{2^{m}} f\left(2^{m} x\right)$ for all $x \in A$. In (6), let $k \rightarrow \infty$, then we get

$$
\left\|\mathrm{f}\left(\frac{n}{n+1} x_{n}\right)-H\left(\frac{n}{n+1} x_{n}\right)\right\| \leq \sum_{i=1}^{\infty} \frac{1}{2^{i}} \varphi\left(0,2^{i-1} x_{2}, \ldots, 2^{i-1} x_{n}, 0, \ldots, 0\right)
$$

for all $x_{2}, \ldots, x_{n-1}, x_{n} \in A$ satisfying $(1+2 n) x_{n}=-\left(x_{2}+\ldots+x_{n-1}\right)$. Since $(1+2 n)\left(\frac{n+1}{n}\right) x_{n}=-\left(\left(\frac{n+1}{n}\right) x_{2}+\ldots+\left(\frac{n+1}{n}\right) x_{n-1}\right)$, thus (5) holds. Now we show that $C_{\mu} H\left(x_{1}, \ldots, x_{n}, 0, \ldots, 0\right)=0$ for all $x_{1}, \ldots, x_{n-1}, x_{n} \in A$ and all $\mu \in \mathbb{C}$ with $|\mu|=1$. Note that $H(0)=0$ and by using (3), we get

$$
\left\|C_{\mu} H\left(x_{1}, \ldots, x_{n}, 0, \ldots, 0\right)\right\| \leq \lim _{m \rightarrow \infty} r^{m} \varphi\left(x_{1}, \ldots, x_{n}, 0, \ldots, 0\right)=0
$$

for all $X \in A^{n}$. In the relation $C_{\mu} H\left(x_{1}, \ldots, x_{n}, 0, \ldots, 0\right)=0$, put $\mu=1, x_{1}=$ $0, w=\frac{n}{n+1} x_{n}$ and $z=\frac{1}{n+1} \sum_{i=2}^{n} x_{i}$, then we get $H(z+w)=H(z)+H(w)$. So, $\mathrm{H}$ is additive. Also, it is now easy to see that $H(\mu x)=\mu H(x)$ for all $\mu \in \mathbb{C}$ with $|\mu|=1$ and all $x$ in $A$. Thus $\mathrm{H}$ is indeed $\mathbb{C}$-linear. To show that $\mathrm{H}$ is an n-ary homomorphism, by using (3), we prove that $C_{\mu} H\left(0, \ldots, 0, y_{1}, \ldots, y_{n}, 0, \ldots, 0\right)=0$ for all $y_{1}, \ldots, y_{n}$ in $A$. We have

$$
\begin{aligned}
\left\|C_{\mu} H\left(0, \ldots, 0, y_{1}, \ldots, y_{n}, 0, \ldots, 0\right)\right\| & \\
& \leq \lim _{m \rightarrow \infty} 2^{m-n m} r^{m} \varphi\left(0, \ldots, 0, y_{1}, \ldots, y_{n}, 0, \ldots, 0\right)=0
\end{aligned}
$$

for all $Y \in A^{n}$. Similarly, $\left\|C_{\mu} H\left(0, \ldots, 0, z_{1}, \ldots, z_{n}, w_{1}, \ldots, w_{n}\right)\right\|=0$, so indeed $H$ is an n-ary Lie homomorphism. 


\section{References}

[1] S.M. Ulam, Problems in Modern Mathematics, John Wiely \& Sons, New York, NY, USA (1964).

[2] D.H. Hyers, On the stability of the linear functional equation, Proceedings of the National Academy of Sciences of the United States of America, 27 (1941), 222-224.

[3] T.M. Rassias, On the stability of the linear mapping in Banach spaces, Proceedings of the American Mathematical Society, 72, No. 2 (1978), 297300.

[4] B. Yousefi, F. Ranjbar, Stability of homomorphisms on some nonArchimedean Lie Banach algebras, Southeast Asian Bulletin of Mathematics (2015), To Appear. 
